\title{
CONTROL OF BIPEDAL LOCOMOTION WITH A NEURAL OSCILLATOR-BASED BRAIN-COMPUTER INTERFACE
}

\author{
${ }^{1}$ Ryo Ikeda \& ${ }^{2}$ Ryota Horie \\ ${ }^{\prime}$ Epson Avasys Corporation, Japan \\ Shibaura Institute of Technology, Japan \\ ma14010@shibaura-it.ac.jp; horie@shibaura-it.ac.jp
}

\begin{abstract}
This study proposes a neural oscillator-based brain-computer interface (BCI) that controls a bipedal neuromusculoskeletal (NMS) model by inputting electroencephalogram (EEG) signals. In this BCI system, while the bipedal NMS system realizes bipedal locomotion through internal entrainment among neural oscillators and a musculoskeletal system, the locomotion of the system is controlled via external entrainment of the neural oscillators to the external input of EEG signals. As the first step in developing the neural oscillator-based BCI controlling a bipedal NMS model, exploratory numerical simulations were conducted to investigate the behavior of the proposed BCI when sinusoidal waves and alpha waves were inputted. The following tendencies were observed: (a) inputting sinusoidal waves with small amplitudes and high frequencies did not affect the natural walking behavior of the bipedal NMS model that was generated by including only offset values in the external input, (b) inputting sinusoidal waves with small amplitudes and low frequencies disturbed and decelerated the walking behavior, (c) inputting sinusoidal waves with large amplitudes accelerated the walking behavior, (d) inputting sinusoidal waves with large amplitudes and a particular frequency changed walking behavior to running behavior, (e) changing the external input of alpha waves between an eyes-open condition and an eyes-closed condition successfully changed the walking behavior. The eyes-open condition led to faster walking compared with the eyes-closed condition.
\end{abstract}

Keywords: bipedal locomotion, brain-computer interface, bipedal neuromusculoskeletal model, entrainment, neural oscillator. 


\section{INTRODUCTION}

Shik, Severin, and Orlovsky (1966) reported that a decerebrated cat started walking when subjected to electrical stimulation to the midbrain. The experiment showed that walking motion is primarily maintained by the function of the lower brain. Central pattern generators (CPGs) are assumed to be at the center of the function. CPGs in spinal nerves autonomously generate rhythmic neuronal activity. Periodic motion is generated by a musculoskeletal system that receives motor commands sent from CPGs.

A mathematical model of neuronal activity in CPGs, called a neural oscillator, was proposed (Matsuoka, 1985, 1987). A neural oscillator generates a stable periodic solution. In addition, a mathematical model of interaction among neural oscillators and a musculoskeletal system, called a bipedal neuromusculoskeletal (NMS) model, was proposed (Taga, Yamaguchi, \& Shimizu, 1991; Taga, 1995). Numerical simulations have shown that the models generate bipedal locomotion as a stable periodic solution for a nonlinear dynamical system. Entrainment among periodic neural activity in the neural oscillators and periodic kinetic patterns in the musculoskeletal system realize bipedal locomotion under appropriately set parameters of the model. Moreover, the model changes gait patterns between walking and running depending on the constant input to the neural oscillators. The changes between the gait patterns occur as a bifurcation phenomenon in the nonlinear dynamical system. The model also shows that walking behavior adaptive to the external environment, such as walking on a slope or recovering from stumbling, occurs through entrainment between the neural oscillators and the musculoskeletal system. More recently, the neural oscillator has been applied to the control of real bipedal (Endo, Nakanishi, Morimoto, \& Cheng, 2005; Matsubara, Morimoto, Nakanishi, Sato, Doya, 2006; Miyakoshi, Taga, Kuniyoshi, \& Nagakubo, 1998), dog-like (Fukuoka \& Kimura, 2009), and snake-like robots (Matsuo \& Ishii, 2007).

On the contrary, there has been growing interest in robot control using a braincomputer interface (BCI) (Choi, \& Jo, 2013). Typical electroencephalogram (EEG)-based BCIs extract specific features from raw EEG signals and discriminate among the features to generate discrete commands, such as on/ off commands based on classification algorithms (Lotte, Congedo, Lecuyer, Lamarche, \& Arnaldi, 2007).

It has been known that nonlinear dynamical systems are entrained to an external rhythmic input. Thus, BCIs to control the locomotion of the robots are expected; it would be dynamically controllable based on neural oscillators 
receiving continuous waves of EEG signals as rhythmic input. Feasibility studies of such BCIs are important because a novel BCI might provide a dynamic, adaptive, and robust control mechanism. Although analyses of rhythmic signals in EEGs observed during locomotion have been studied (Castermans, Duvinage, Cheron, \& Dutoit, 2014), neural oscillator-based BCIs have been studied less (Ikeda \& Horie, 2014; Horie, Ikeda, Otsuka \& Hashimoto, 2016).

Some studies have proposed hybrid systems combining a BCI and neural oscillators (Gui, Ren, \& Zhang, 2010; Jia, Huang, Luo, Pu, Chen, \& Bai, 2012; Zhang, Liu, Huan, Liu, \& Zhu, 2009). In the systems, a BCI generates discrete commands and the commands changed a mode of neural oscillator-based locomotion. The neural oscillators don't receive continuous EEG signals.

This study proposes a novel BCI that controls a bipedal NMS model by inputting EEG signals. Figure 1 shows a conceptual diagram of proposed neural oscillator-based BCI. While the bipedal NMS system realizes bipedal locomotion via internal entrainment among the neural oscillators and the musculoskeletal system, the system locomotion is controlled via external entrainment of the neural oscillators to the externally inputted EEG signals.

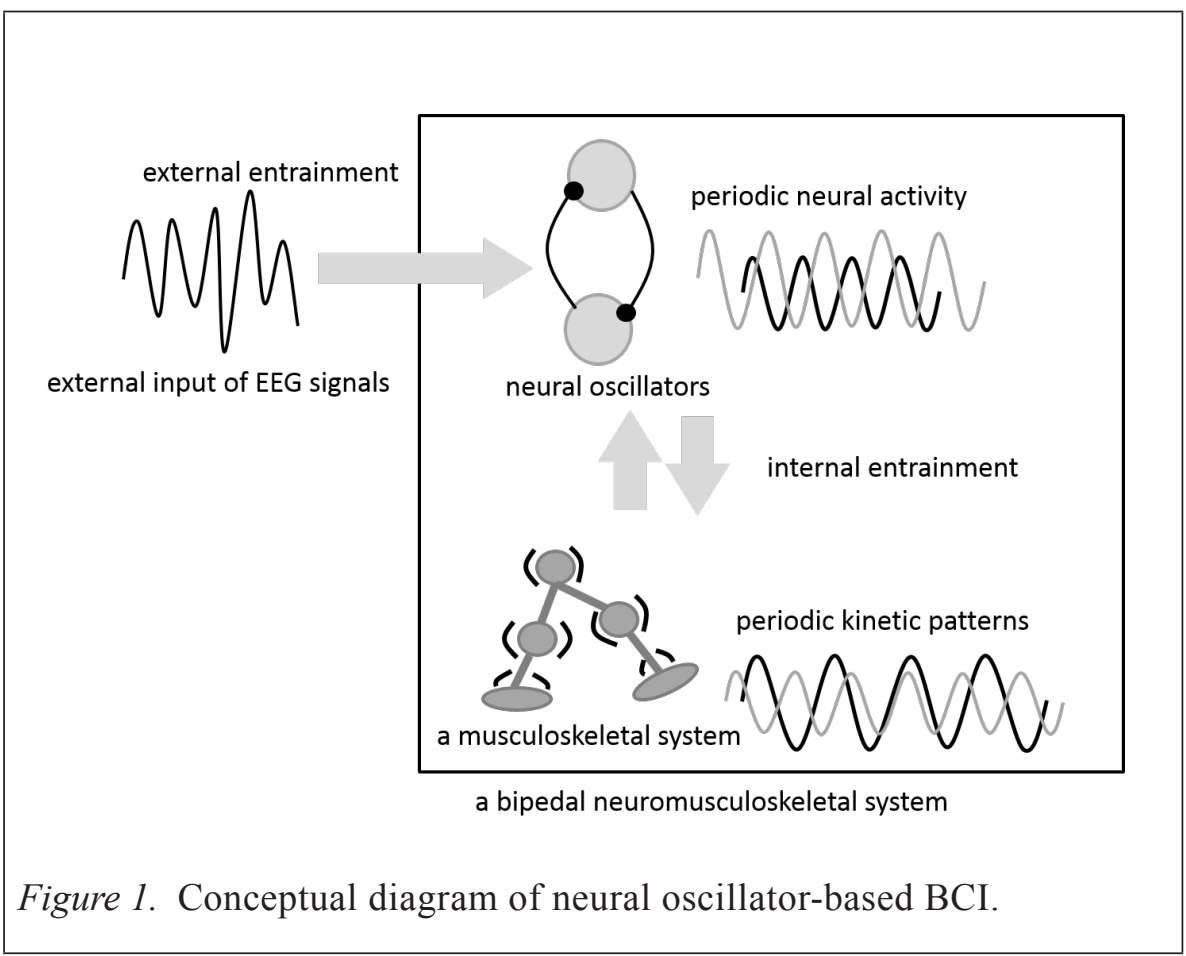


This preliminary study revealed that motion patterns of a pendulum, the simplest NMS system, were successfully modulated by the change of external inputting of alpha waves between eyes-open and eyes-closed conditions (Ikeda \& Horie, 2014). This study is the first step in developing the neural oscillator-based BCI controlling a bipedal NMS model. The idea of the proposed model and the preliminary results were partially reported (Horie et al., 2016). In this paper, a wholly empirical approach was taken to reveal the feasibility of the neural oscillator-based BCI while the theoretical interpretation of the entrainment phenomena has been widely studied (Glass, 2001; Pikovsky, Rosenblum, \& Kurths, 2001). The behavior of the proposed BCI was investigated when sinusoidal waves and alpha waves were inputted by conducting exploratory numerical simulations.

\section{MATHEMATICAL MODEL}

Figure 2(a) shows Matsuoka's neural oscillator model (Matsuoka, 1985, 1987). In the model, one oscillator is composed of a pair of dynamical neurons. Each neuron is modeled by two-dimensional (2D) ordinary differential equations. Figure 2(b) shows Taga's bipedal NMS model (Taga et al., 1991), which is composed of six of Matsuoka's neural oscillators and a 2D musculoskeletal system of the lower body. The six neural oscillators correspond to hip, knee, and ankle units on the left and right sides. In this study, Taga's model was used as a control target of the BCI. The model of the six neural oscillators was represented by 12 pairs of ordinary differential equations, as follows:

$$
\begin{aligned}
& \tau_{i} \dot{u}_{i}(t)=-u_{i}(t)+\sum_{j=1}^{12} w_{i j} y_{j}(t)-\beta v_{i}(t)+u_{0}(t)+\text { Feed }_{i}(t), \quad i=1, \ldots, 12 \\
& \dot{\tau}_{i} \dot{v}_{i}(t)=-v_{i}(t)+y_{i}(t), \quad i=1, \ldots, 12 \\
& y_{i}=\max \left(0, u_{i}\right), \quad i=1, \ldots, 12
\end{aligned}
$$

where $u_{i}$ is the inner state of the $i^{\text {th }}$ neuron, $v_{i}$ is a variable representing the degree of adaptation or self-inhibition of the $i^{\text {th }}$ neuron, $\beta$ is the strength of the adaptation, $y_{i}$ is the output of the $i^{\text {th }}$ neuron, $w_{i j}$ is the connecting weight between the $i^{\text {th }}$ and $j^{\text {th }}$ neurons, $\tau$ and $\tau$ are time constants of the inner state and adaptation effect, respectively. Feed $(\mathrm{t})$ is a sensory feedback signal to the $i^{\text {th }}$ neuron from the musculoskeletal system, and $u_{0}(t)$ is the external input to the $i^{\text {th }}$ neuron. A unique external input $u_{\mathrm{o}}(t)$ is inputted to all 12 neurons. In this study, the ordinary differential equations and values of their parameters were 
the same as in Taga's model [4], except that the external input $u_{0}(t)$ was time dependent. In this proposed $\mathrm{BCI}$, the external input was provided using EEG signals.

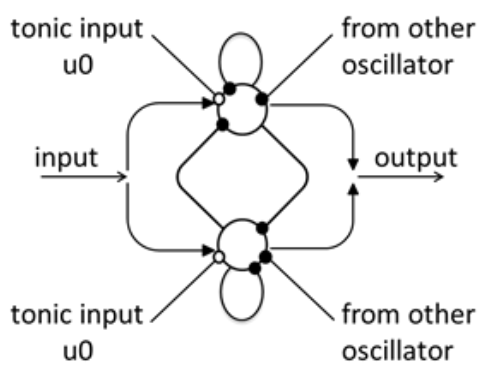

(a) Matsuoka's neural oscillator.

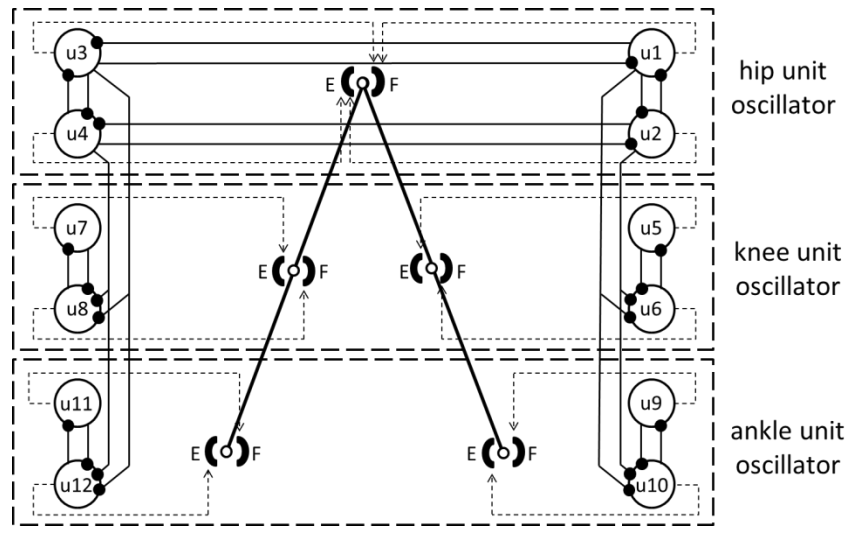

(b) Taga's bipedal NMS model.

Figure 2. Neural oscillator and bipedal NMS model.

The kinetic model of the musculoskeletal system, not shown in this paper, and their parameters were the same as in Taga's model (Taga et al., 1991), wherein the equations of motion for the musculoskeletal system were derived using the Newton-Euler method. Interactions between the neural oscillators and the musculoskeletal system, and its parameters were also the same as in Taga's model (Taga et al., 1991). The torque acting at the joints is induced by the output of neural oscillators. The states of the joint angles determine the feedback signal inputted to the neural oscillators. 


\section{METHODOLOGY}

This section presents the method used in the study. This includes the descriptions of numerical simulation, sinusoidal waves as input and the experimental protocol to measure EEG.

\section{Numerical Simulation}

Taga's model, explained above, was reconstructed using Simulink/ SimMechanics from Mathworks Inc. The initial conditions of the bipedal NMS model were the same as in Taga's study (Taga et al., 1991). The ground on which the musculoskeletal system walked was a horizontal floor. Using the NMS model, bipedal locomotion was simulated for $60 \mathrm{~s}$. The number of steps, average stride length $[\mathrm{m}]$, and moving distance $[\mathrm{m}]$ in the locomotion were calculated. All indices were measured during the $60 \mathrm{~s}$. In addition, to identify whether a gait pattern was walking or running, it was investigated whether the number of jumps becomes equal to the number of steps. When a gait pattern becomes running, the number of jumps becomes equal to the number of steps because of the presence of the flight phase. In contrast, when a gait pattern is walking, the number of jumps becomes less than the number of steps (ideally zero) because of the presence of the double-support phase. At the beginning of a simulation, the model converged to a steady walking/running within a few cycles of walking/running. Transient behavior was removed from the analyses.

\section{Sinusoidal Waves as Input}

To obtain basic knowledge of how the model behaves according to the time-dependent external input $u_{0}(t) u_{0}(t)$ to the neural oscillators, motion patterns of the model were simulated when various types of sinusoidal waves were inputted as the external input, and examined the resulting behavior of the model. The amplitude of the sinusoidal wave was set to 5 or 30 . The frequency of the sinusoidal wave was changed from $1 \mathrm{~Hz}$ up to $50 \mathrm{~Hz}$ in steps of $1 \mathrm{~Hz}$. In the external input, an offset value of 6.5 was added to the sinusoidal waves. When the external input is only the offset without the sinusoidal wave, the system becomes the original bipedal NMS model investigated in Taga's study (Taga et al., 1991).

\section{EEG Experimental Protocol}

EEG signals were measured while a participant was relaxed in both the eyes-open and eyes-closed conditions, each for $60 \mathrm{~s}$. It has been known that EEG changes depending on human mental state and activity. Alpha waves 
(8-13 Hz) markedly appear when one relaxes or closes one's eyes. One reason that both the eyes-open and eyes-closed conditions were used was to obtain clear changes in the increase and decrease of the alpha waves. In the first step of investigating how EEG signals affect the bipedal NMS model, clear changes in EEG signals must be used, even though the changes between the two conditions had no practical meaning in the context of controlling a bipedal robot using BCI.

In the experiment to measure EEG, an electrode was attached to each participant's scalps at the $\mathrm{Pz}$ sites of the International 10-20 system. The electrode was referenced to the left mastoid. Impedance at the electrode sites was less than $5 \mathrm{k} \Omega$. The EEG was amplified by a DC amplifier (Melon Technos Co. Ltd., $8 \mathrm{ch}$ amplifier) with a gain of 50,000, a 1-30-Hz bandpass filter, and a $50-\mathrm{Hz}$ notch filter, and was continuously sampled at $1,000 \mathrm{~Hz}$ in 16 bits using a data recorder (NF Corporation, EZ7510).

\section{Inputting EEG Signals}

Motion patterns of the model were simulated when the EEG signals were inputted as the external input to the neural oscillators. The measured EEG signals were passed through an offline finite impulse response bandpass filter $(8-13 \mathrm{~Hz})$ to extract the alpha-band waves. As the external input to the neural oscillators, the filtered EEG signal was inputted to all oscillators with a commonly defined gain. The gain for the alpha wave was changed from one up to a gain at which the bipedal NMS fell down in steps of one. In the external input, the offset value of 6.5 was added to the alpha waves. Behaviors with the alpha wave inputted in the eyes-open and eyes-closed conditions were compared.

\section{RESULTS AND DISCUSSION}

Exploratory numerical experiments have been performed to investigate the behavior of the proposed BCI. This section presents the simulated walking behavior of the bipedal NMS model when sinusoidal waves with small amplitude, sinusoidal waves with large amplitude, and EEG signals were input.

\section{Results of Inputting Sinusoidal Waves with Small Amplitude}

Figure 3 shows the results of the simulations wherein the sinusoidal waves were inputted to the model with the external input and amplitude of the sinusoidal waves set to five as a small amplitude. The vertical axes of the 
four graphs, (a), (b), (c), and (d), of Figure 3 indicate the number of steps, average stride lengths $[\mathrm{m}]$, moving distance $[\mathrm{m}]$, and number of steps and jumps, respectively, indicating a gait pattern. The horizontal axes of the graphs indicate the frequency of the sinusoidal wave used for the external input. In the top three graphs, (a), (b), and (c), dashed lines indicate results when only the offset value of 6.5 was inputted as the external input. In graph (d), black cross marks and lines connecting them indicate the number of steps, which is the same as that in graph (a), while gray cross marks and lines connecting them indicate the number of jumps. In the four graphs, the absence of cross marks indicates that the model fell down from inputting the sinusoidal wave of the specified frequency.

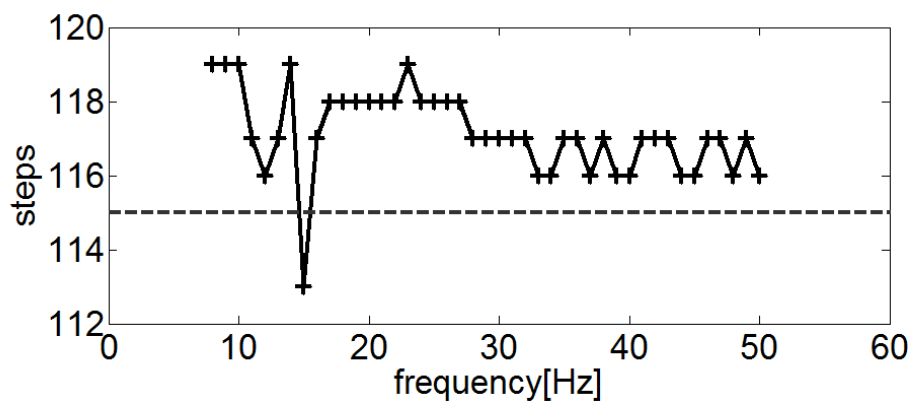

(a) Numbers of steps.

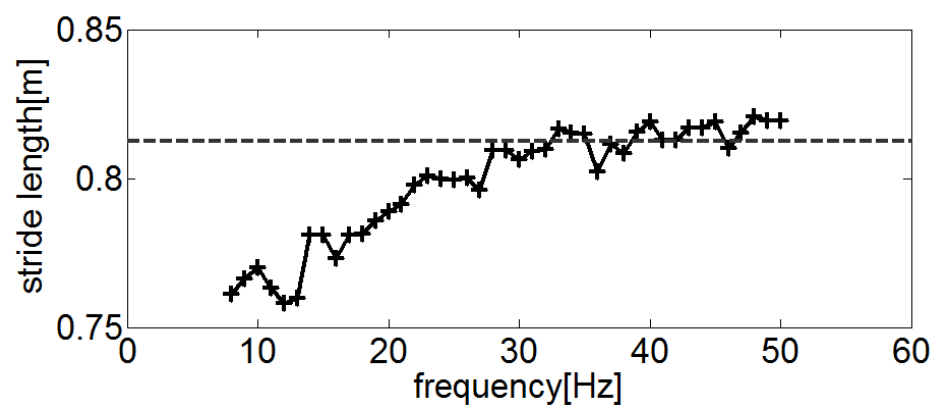

(b) Average stride lengths. 


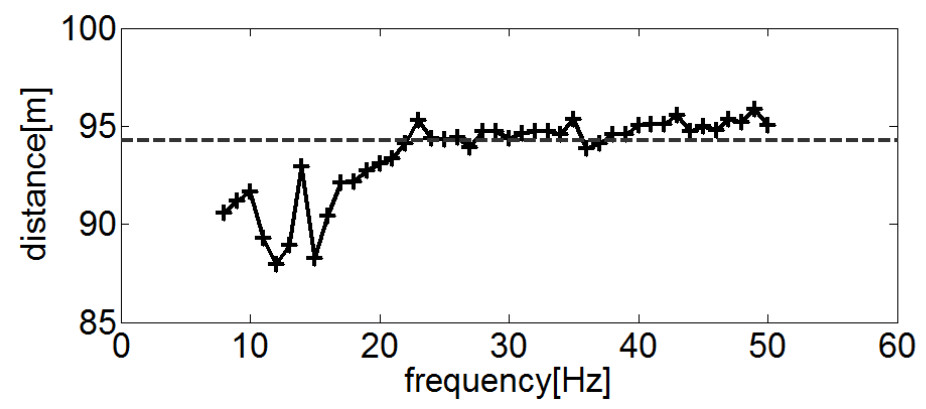

(c) Moving distances.

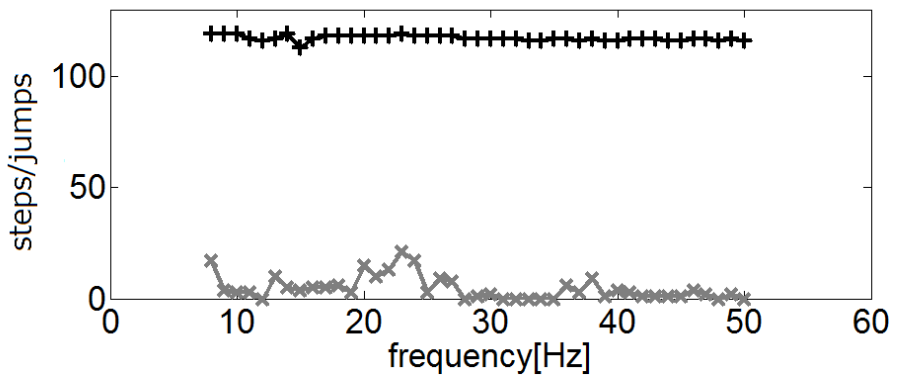

(d) Moving distances.

Figure 3. Inputting sinusoidal waves with small amplitude.

In graphs (b) and (c), the average stride lengths and moving distance had a tendency to increase when the frequency of the sinusoidal wave increased. When the frequency of the sinusoidal wave was greater than approximately $30 \mathrm{~Hz}$, the two indices converged to the values that were acquired when only the offset value was inputted. Graph (d) shows that every gait pattern was walking. In addition, the model fell down when the frequency of the sinusoidal wave was less than $8 \mathrm{~Hz}$.

The results show an overall tendency for the model locomotion to be unaffected by the input of the sinusoidal wave when the frequency of the sinusoidal wave increases (above approximately $30 \mathrm{~Hz}$ ). It is assumed that the locomotion was entrained to the natural behavior that the system generates with only the offset value. The external input with the small amplitude (amplitude of 5) might have 
a weak influence. On the contrary, when the frequency of the sinusoidal wave was less (less than approximately $30 \mathrm{~Hz}$ ), the speed of walking became slower than the speed of the natural behavior. It is assumed that the natural behavior was disturbed and decelerated by the external input of the low-frequency sinusoidal wave even when the amplitude of the sinusoidal wave was small.

\section{Result of Inputting Sinusoidal Waves with Large Amplitude}

Figure 4 shows results of the simulations wherein the sinusoidal waves were inputted to the model as the external input, and amplitude of the sinusoidal waves was set to 30 as a large amplitude. The vertical and horizontal axes in the four graphs, (a), (b), (c), and (d), the dashed lines in the top three graphs, (a), (b), and (c), and the black/gray cross marks and lines connecting them in graph (d) are the same as that in Figure 3, respectively. In the four graphs, the absence of cross marks indicates that the model fell down as a result of inputting the sinusoidal waves of the frequency.

In the graphs (b) and (c), the average of stride lengths and moving distance decreased when the frequency of the sinusoidal wave increased. The graph (d) shows that the gait pattern changed suddenly from walking to running when the frequency of the sinusoidal wave became $29 \mathrm{~Hz}$. In addition, the model fell down when the frequency of the sinusoidal wave was $21 \mathrm{~Hz}, 23 \mathrm{~Hz}, 25 \mathrm{~Hz}$, and less than $20 \mathrm{~Hz}$.

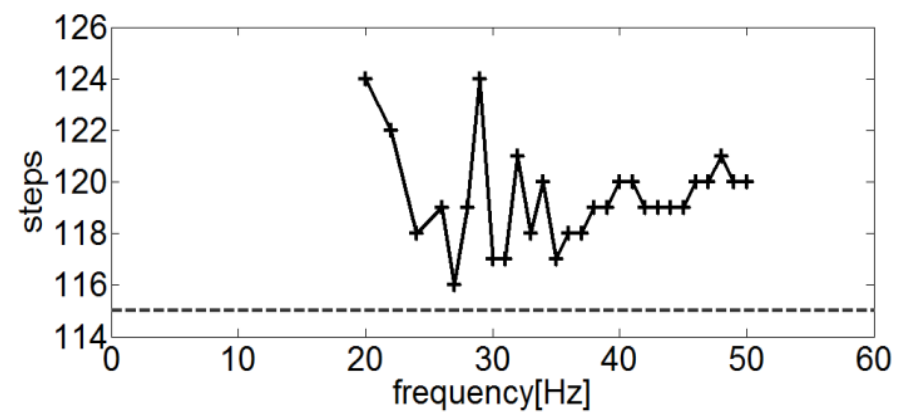

(a) Numbers of steps. 


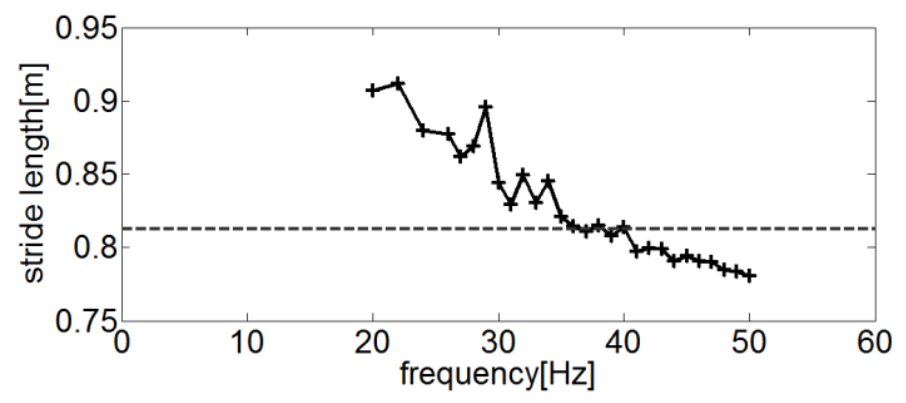

(b) Average stride lengths.

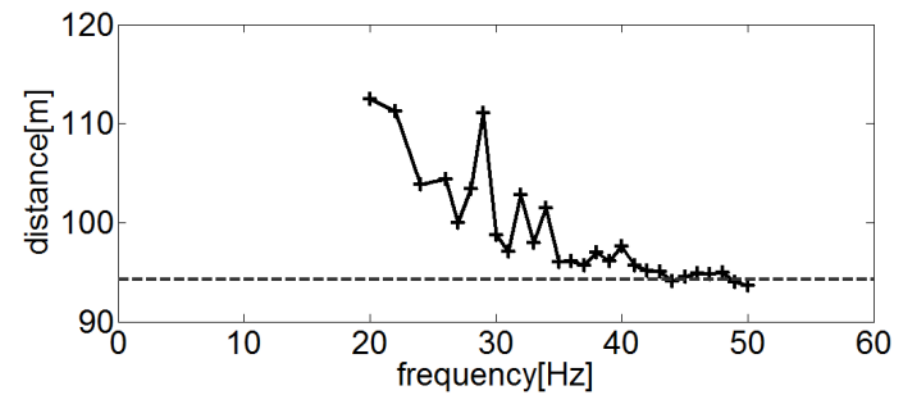

(c) Moving distances.

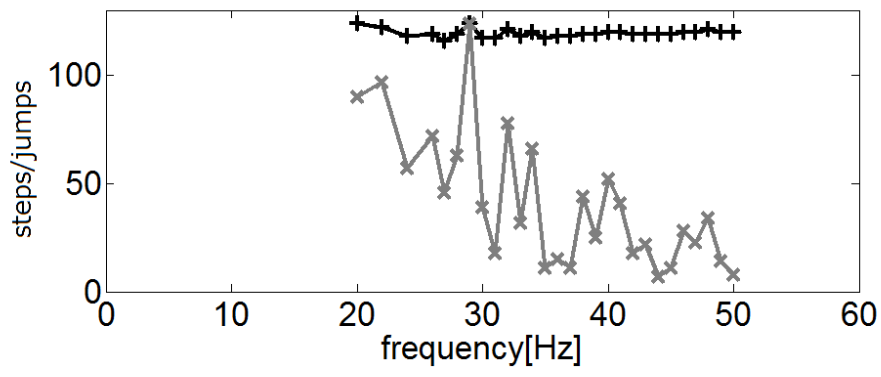

(d) Gait patterns

Figure 4. Inputting sinusoidal waves with large amplitude. 
The results show an overall tendency for the locomotion of the model to be affected by the input of the sinusoidal wave in the entire frequency range of the sinusoidal wave. When that frequency was less (less than approximately $40 \mathrm{~Hz}$ ), the speed of walking became faster than the speed of the natural behavior that the system generates only with the offset value. It is assumed that the locomotion was entrained to the external input with the large amplitude (amplitude of 30) and accelerated. Additionally, the results show a sudden change from walking to running for a particular frequency $(29 \mathrm{~Hz})$. It is suspected that the sudden change might be a type of nonlinear resonance.

\section{Controlling Bipedal Locomotion by Inputting EEG Signals}

The results above show that the locomotion behavior of the model was decelerated and accelerated when the low-frequency sinusoidal wave with the small and large amplitudes, respectively, were inputted to the model. Thus, it was expected that a low-frequency EEG signal might control the locomotion of the model depending on its amplitude. Consequently, the change in the locomotion behavior was investigated when an alpha wave used as a lowfrequency wave was inputted to the model and the amplitude of the alpha wave increased and decreased. The increase and decrease of the amplitude were caused by changing the gain of the external input and changing between the eyes-open and eyes-closed conditions.

\section{Result of Inputting EEG Signals}

Figures 5 shows the results of simulations wherein the alpha waves were inputted as the external input. The vertical axes of the five panels, (a), (b), (c), (d) and (e), of Figure 5 indicate the number of steps, average stride lengths $[\mathrm{m}]$, moving distance [m], and number of steps and jumps in the eyesopen condition, indicating a gait pattern, and in the eyes-closed condition, respectively. All indices were measured within $60 \mathrm{~s}$. The horizontal axes of the graphs indicate the gain for the alpha wave. In the top three graphs, (a), (b), and (c), the black cross marks and lines connecting them, the gray cross marks and lines connecting them, and the dashed lines, indicate the indices acquired in the eyes-open condition, in the eyes-closed condition, and when only the offset value of 6.5 was inputted. In graphs (d) and (e), the black cross marks and lines connecting them indicate the number of steps, just as in the graph (a), while gray cross marks and lines connecting them indicate the number of jumps. In the five graphs, the absence of cross marks indicates that the model fell down from inputting the alpha wave with the gain. 


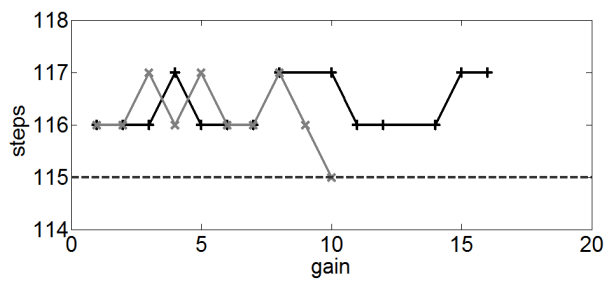

(a) Numbers of steps.

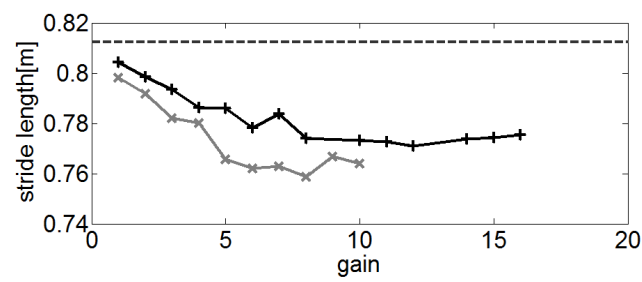

(b) Average stride lengths.

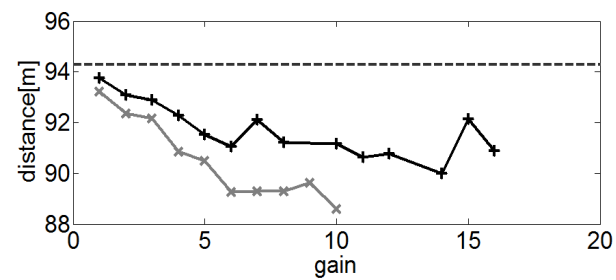

(c) Moving distances.

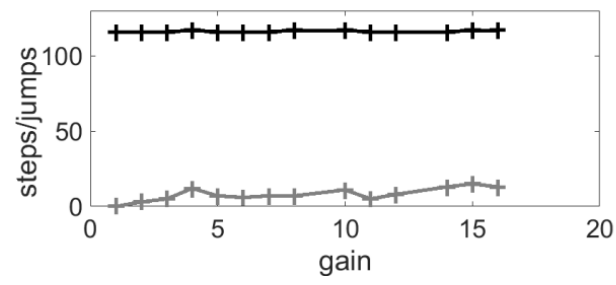

(d) Gait patterns in eyes-open condition.

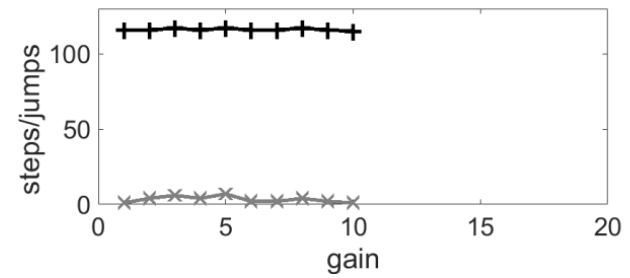

(e) Gait patterns in eyes-closed condition.

Figure 5. Result of inputting EEG signals. 
In graphs (b) and (c), the average of stride lengths and moving distance decreased when the gain of the alpha wave increased. The figures show that the alpha wave in the eyes-closed condition caused lower average stride lengths and shorter moving distances than those acquired in the eyes-open condition. The model kept walking in a wider range of the gain (gain from one to eight, from 10 to 12, and 17), while the model fell down in a range of high gain (gain above 10) in the eyes-closed condition. In both conditions, walking became slower than the speed of the natural behavior that the system generates with only the offset value. Graphs (d) and (e) show that every gait pattern was walking.

The results indicate that walking behavior was successfully changed by the change of external input of the alpha waves between the eyes-open and eyesclosed conditions. The eyes-open condition caused faster walking compared with the eyes-closed condition. The change was obtained when the bipedal NMS model did not fall down in both conditions. The gain of the alpha waves that satisfy the requirement was in the range of one to eight or set to ten. A relatively large change of walking behavior was obtained when the gain was in the range of approximately five to eight or set to ten.

It is assumed that the mechanism of controlling walking behavior by inputting alpha waves was similar to that of inputting sinusoidal waves with a small amplitude (amplitude of 3). It is widely known that the amplitude of alpha waves increases in the eyes-closed condition in comparison with the eyesopen condition. Thus, the large amplitude of the alpha wave caused in the eyes-closed condition disturbed and decelerated the natural behavior that the system generates with only the offset value. The disturbance was strong when the gain of the alpha wave increased.

Another controlling mechanism similar to the case of inputting sinusoidal waves with large amplitude (amplitude of 30) was expected. In that mechanism, locomotion might be accelerated by low-frequency EEG signals such as alpha waves. However, the model fell down when the gain of the alpha wave increased, as shown in the results obtained in this study.

\section{Motion Trace of the Simulated Walking Behavior}

Figure 6 shows the typical walking behavior plotted by a motion trace of the simulated model in steps of $0.1 \mathrm{~s}$. Figure 6(a) illustrates the natural walking behavior when only the offset value of 6.5 was inputted as the external input. Figure 6(b) illustrates the walking behavior when the sinusoidal wave with a 
small amplitude (amplitude of 5) and a low frequency (10 Hz) was inputted. Figure 6(c) illustrates running behavior when the sinusoidal wave with a large amplitude (amplitude of 30) and a special frequency of $29 \mathrm{~Hz}$, which induced the running behavior, were inputted. Figure 6(d) illustrates the walking behavior when the alpha wave was inputted with a gain of ten. In the fourmotion trace, arrows indicate steps of $1.0 \mathrm{~s}$.

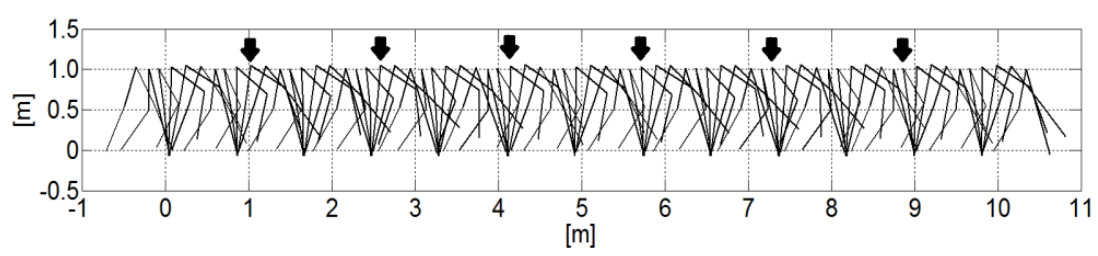

(a) Inputting only offset value.

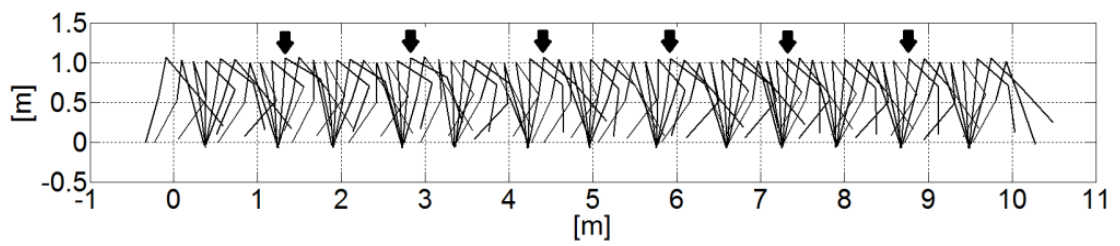

(b) Inputting a sinusoidal wave with small amplitude.

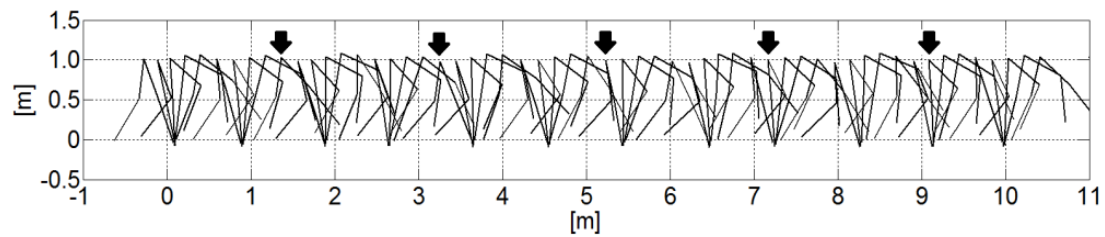

(c) Inputting a sinusoidal wave with large amplitude.

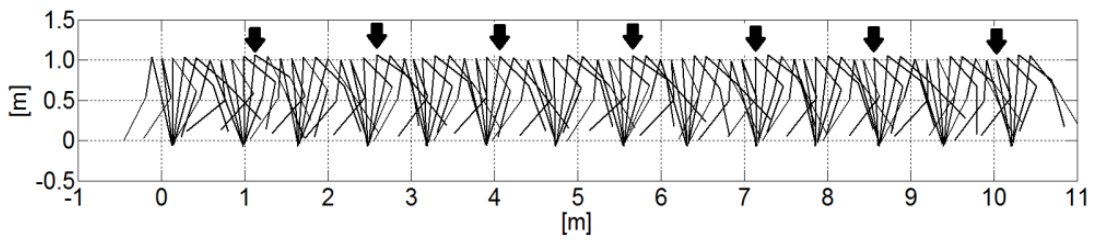

(d) Inputting an EEG signal.

Figure 6. Motion trace of the simulated walking behavior. 
The walking behavior for inputting the sinusoidal wave with a small amplitude, illustrated in Figure 6(b), was slower than the natural walking behavior, illustrated in Figure 6(a). The running behavior for inputting the sinusoidal wave with a large amplitude, illustrated in Figure 6(c), was faster than the natural walking behavior.

The walking behavior for inputting the alpha wave, illustrated in Figure 6(d), had fluctuations in its strides, as observed in the real walking behavior (Dingwell \& Cusumano, 2010), in comparison with the walking behavior for inputting the sinusoidal waves and natural walking behavior. Fluctuation in the alpha wave might cause fluctuation in the walking behavior. It is speculated that the bipedal NMS model for EEG signals being inputted can be used for the modeling of real walking behavior.

\section{CONCLUSION}

This paper has proposed a neural oscillator-based BCI that controls a bipedal NMS model by inputting EEG signals. In the BCI system, while the bipedal NMS system realizes bipedal locomotion through internal entrainment among the neural oscillators and musculoskeletal system, the locomotion of the bipedal NMS system is controlled via external entrainment of the neural oscillators to externally inputting EEG signals. The behavior of the proposed BCIs, when sinusoidal and alpha waves were inputted, was investigated by conducting exploratory numerical simulations. The following tendencies were found: (a) inputting sinusoidal waves with a small amplitude and a high frequency did not affect the natural walking behavior of the bipedal NMS model that was generated by only the offset value, (b) inputting sinusoidal waves with a small amplitude and a low frequency disturbed and decelerated the walking behavior, (c) inputting sinusoidal waves with a large amplitude accelerated the walking behavior, (d) inputting sinusoidal waves with a large amplitude with a particular frequency caused running behavior, and (e) walking behavior was successfully changed by a change of the external input of the alpha waves between the eyes-open and eyes-closed conditions. The eyes-open condition generated faster walking compared with the eyes-closed condition.

Future studies, need to evaluate the reproducibility of the results with more subjects and using a real-time system. The control of walking behavior by inputting alpha waves, in this study, was realized by the deceleration of the natural behavior. There is the possibility of realizing control mechanisms for 
acceleration of walking and transition to running by inputting EEG signals, as found in the case of inputting sinusoidal waves with large amplitudes. The final goal is to develop EEG-based BCIs that control bipedal locomotion of robots based on human mental states related to lower limb motion.

\section{REFERENCES}

Castermans, T., Duvinage, M., Cheron, G., \& Dutoit, T. (2014). About the cortical origin of the low-delta and high-gamma rhythms observed in EEG signals during treadmill walking. Neuroscience Letters, 561, 166170. doi: 10.1016/j.neulet.2013.12.059

Choi, B., \& Jo, S. (2013). A low-cost EEG system-based hybrid brain-computer interface for humanoid robot navigation and recognition. PLOS ONE, 8(9), e74583. doi:10.1371/journal.pone.0074583

Dingwell, J.B., \& Cusumano, J.P. (2010). Re-interpreting detrended fluctuation analyses of stride-to-stride variability in human walking. Gait \& Posture, 32(3), 348-353. doi: 10.1016/j.gaitpost.2010.06.004

Endo, G., Nakanishi, J., Morimoto, J., \& Cheng, G. (2005). Experimental studies of a neural oscillator for biped locomotion with QRIO. Proceedings of the IEEE International Conference on Robotics and Automation, 2005 (ICRA 2005). (pp. 596-602). IEEE. doi: 10.1109/ ROBOT.2005.1570183

Fukuoka, Y., \& Kimura, H. (2009). Dynamic locomotion of a biomorphic quadruped 'Tekken' robot using various gaits: walk, trot, free-gait and bound. Applied Bionics and Biomechanics, 6(1), 63-71. doi: 10.1080/11762320902734208

Glass, L. (2001). Synchronization and rhythmic processes in physiology. Nature, 410, 277-284. doi:10.1038/35065745

Gui, K., Ren, Y., \& Zhang, D. (2010). Online brain-computer interface controlling robotic exoskeleton for gait rehabilitation. 2015 IEEE International Conference on Rehabilitation Robotics (ICORR). (pp. 931-936). IEEE. doi:10.1109/ICORR.2015.7281323 
Horie, R., Ikeda, R., Otsuka, Y., \& Hashimoto, T. (2016, August). A bipedal neuromusculoskeletal model with input of EEG. Paper presented at the 38th Annual International Conference of the IEEE Engineering in Medicine and Biology Society (EMBC'16), Orlando. Retrieved from http://embc.embs.org/2016/embc-2016-proceedings

Ikeda, R., \& Horie, R. (2014, August). Toward neural oscillator based braincomputer interface. Paper presented at the 36th Annual International Conference of the IEEE Engineering in Medicine and Biology Society (EMBC'14), Chicago. Retrieved from http://emb.citengine.com/event/ embc-2014/details

Jia, W., Huang, D., Luo, X., Pu, H., Chen, X., \& Bai, O. (2012). Electroencephalography (EEG)-based instinctive brain-control of a quadruped locomotion robot. 2012 Annual International Conference of the IEEE Engineering in Medicine and Biology Society (EMBC). (pp. 1777-1781). IEEE. doi:10.1109/EMBC.2012.6346294

Lotte, F., Congedo, M., Lecuyer, A., Lamarche, F., \& Arnaldi, B. (2007). A review of classification algorithms for EEG-based braincomputer interfaces. Journal of Neural Engineering, 4(2). R1-R13. doi:10.1088/1741-2560/4/2/R01

Matsubara, T., Morimoto, J., Nakanishi, J., Sato. M., \& Doya, K. (2006). Learning CPG-based biped locomotion with a policy gradient method. Robotics and Autonomous Systems, 54(11), 911-920. doi: 10.1016/j. robot.2006.05.012

Matsuo, T., \& Ishii, K. (2007). Neural oscillator based motion control system for snake-like robot. Symposium on Underwater Technology and Workshop on Scientific Use of Submarine Cables and Related Technologies, 2007. (pp. 397-402). IEEE. doi: 10.1109/UT.2007.370749

Matsuoka, K. (1985). Sustained oscillations generated by mutually inhibiting neurons with adaptation. Biological Cybernetics, 52(6), 367-376. doi: 10.1007/BF00449593

Matsuoka, K. (1987). Mechanisms of frequency and pattern control in the neural rhythm generators. Biological Cybernetics, 56(5), 345-353. doi:10.1007/BF00319514 
Miyakoshi, S., Taga, G., Kuniyoshi, Y., \& Nagakubo, A. (1998). Three dimensional bipedal stepping motion using neural oscillators humanoid motion in the real world. 1998 IEEE/RSJ International Conference on Intelligent Robots and Systems, 1998. Proceedings, 1. (pp. 84-89). doi:10.1109/IROS.1998.724601

Pikovsky, A., Rosenblum, M., \& Kurths., J. (2001). Synchronization: A universal concept in nonlinear sciences. Cambridge, New York, Melbourne, Madrid, Cape Town, Singapore, São Paulo: Cambridge University Press.

Shik, M.L., Severin, F.V., \& Orlovsky, G.N. (1966). Control of walking and running by means of electrical stimulation of the midbrain. Biophysics, 11, 756-765.

Taga, G., Yamaguchi, Y., \& Shimizu, H. (1991). Self-organized control of bipedal locomotion by neural oscillators in unpredictable environment. Biological Cybernetics, 65(3), 147-159. doi:10.1007/BF00198086

Taga, G. (1995). A model of the neuro-musculo-skeletal system for human locomotion: I. Emergence of basic gait. Biological Cybernetics, 73(2), 97-111. doi:10.1007/BF00204048

Zhang, D., Liu, G., Huan, G., Liu, J., \& Zhu, X. (2009). A hybrid FES rehabilitation system based on $\mathrm{CPG}$ and $\mathrm{BCI}$ technology for locomotion: A preliminary study. In M. Xie, Y. Xiong, C. Xiong, H. Liu, Z. Hu (Eds.). Intelligent robotics and applications: Second International Conference, ICIRA 2009, Singapore, December 16-18, 2009, Proceedings, Lecture Notes in Computer Science, 5928. (pp. 1073-1084). Berlin: SpringerVerlag. doi:10.1007/978-3-642-10817-4_105 